\title{
LETTER \\ Object Extraction Using an Edge-Based Feature for Query-by-Sketch Image Retrieval
}

\author{
Takuya TAKASU $^{\dagger \text { a)}}$, Yoshiki KUMAGAI ${ }^{\dagger \dagger}$, Nonmembers, and Gosuke OHASHI ${ }^{\dagger}$, Member $^{2}$
}

\begin{abstract}
SUMMARY We previously proposed a query-by-sketch image retrieval system that uses an edge relation histogram (ERH). However, it is difficult for this method to retrieve partial objects from an image, because the ERH is a feature of the entire image, not of each object. Therefore, we propose an object-extraction method that uses edge-based features in order to enable the query-by-sketch system to retrieve partial images. This method is applied to 20,000 images from the Corel Photo Gallery. We confirm that retrieval accuracy is improved by using the edge-based features for extracting objects, enabling the query-by-sketch system to retrieve partial images. key words: query-by-sketch image retrieval, object extraction, partial image retrieval, edge-based feature
\end{abstract}

\section{Introduction}

Recently, large-capacity storage media and digital media devices such as PCs and digital cameras have become common, and networks have become faster. This has led to a demand for image retrieval systems capable of processing a large volume of images [1]. Against such a backdrop, there have been various studies of systems for content-based image retrieval (CBIR) [2]-[9]. In CBIR, image features such as color, shape, and texture are used along with queries in the form of examples and sketched images. Query-bysketch image retrieval is efficient, and the queries are simple. It can also be used when there is no example of the desired image at hand. Many these systems employ global descriptors [3]-[8]. On the other hand, there are some systems that employ local features [9], [10]. It is possible to conduct partial image matching by using local features, but it is difficult to conduct approximate image matching by rough sketches. It is written that "in CBIR the goals are somewhat different: the aim is to retrieve semantically-relevant images, even if they do not appear to be visually similar" on the reference [10]. We have already proposed a queryby-sketch image retrieval method that uses an edge relation histogram (ERH), which is a feature that focuses on the relationships between the binary edge images [7], [8]. This system is not affected by the position, size, rotation, or mirroring of the images. However, partial image retrieval using

\footnotetext{
Manuscript received July 17, 2014.

Manuscript revised September 24, 2014.

Manuscript publicized October 15, 2014.

$\dagger$ The authors are with the Department of Electrical and Electronic Engineering, Shizuoka University, Hamamatsu-shi, 4328561 Japan.

${ }^{\dagger}$ The author is with Nidek Co. Ltd., Gamagori-shi, 443-0038 Japan.

a) E-mail: f0330119@ipc.shizuoka.ac.jp DOI: 10.1587/transinf.2014EDL8147
}

the ERH is difficult, because the ERH is a feature obtained from the entire image. It is thus necessary to extract the feature from each object in order to enable partial image retrieval. There is labeling to extract linking components of pixels, as a method of dividing up a binary image, but an object configured from a number of edge curves cannot be extracted, so it is difficult to apply this as object extraction. Therefore, this study proposes the extraction of objects using edge-based features, with the objective of enabling partial retrieval in a query-by-sketch image retrieval system.

\section{Query-by-Sketch Image Retrieval}

Figure 1 shows an overview of the query-by-sketch partial image retrieval system. In query-by-sketch image retrieval, the system calculates the similarity between the features of a sketch image and the features in a database of edge images; the candidate images are then output in order of decreasing similarity. In order to retrieve only part of an image, it is necessary to extract an object from the edge images of the database images. The proposed object extraction method is based on the edge-based features, which are obtained during the ERH extraction. We extract the ERH from each object in each image, and use this as a feature of the image from the database. The similarity between the sketch and each database image is used as a measure of the maximum value of the similarity between the sketch and each object. Candidate images are then displayed in order of decreasing similarity.

\section{Object Extraction Using the Global Distribution of an Edge-Based Feature}

The global edge pixel distribution obtained by the ERH extraction mechanism is centered on each edge pixel and is handled as an edge-based feature for each edge pixel; it takes a value similar to that of nearby pixels. The object extraction is then based on the degree of similarity between the features with respect to each edge pixel. Figure 2 shows radar charts for the global edge pixel distributions obtained from each pixel of interest. The global edge pixel distributions in Figs. 2(a-1) to 2(a-4) are shown in Figs. 2(b-1) to 2(b-4), respectively. Figures 2(a-1) to 2(a-3) show cases where the pixel of interest was moved to an adjacent pixel on the same object, whereas Fig. 2(a-4) shows a case in which the pixel of interest was treated as an edge pixel of a different object from that used in Figs. 2(a-1) to 2(a-3). 


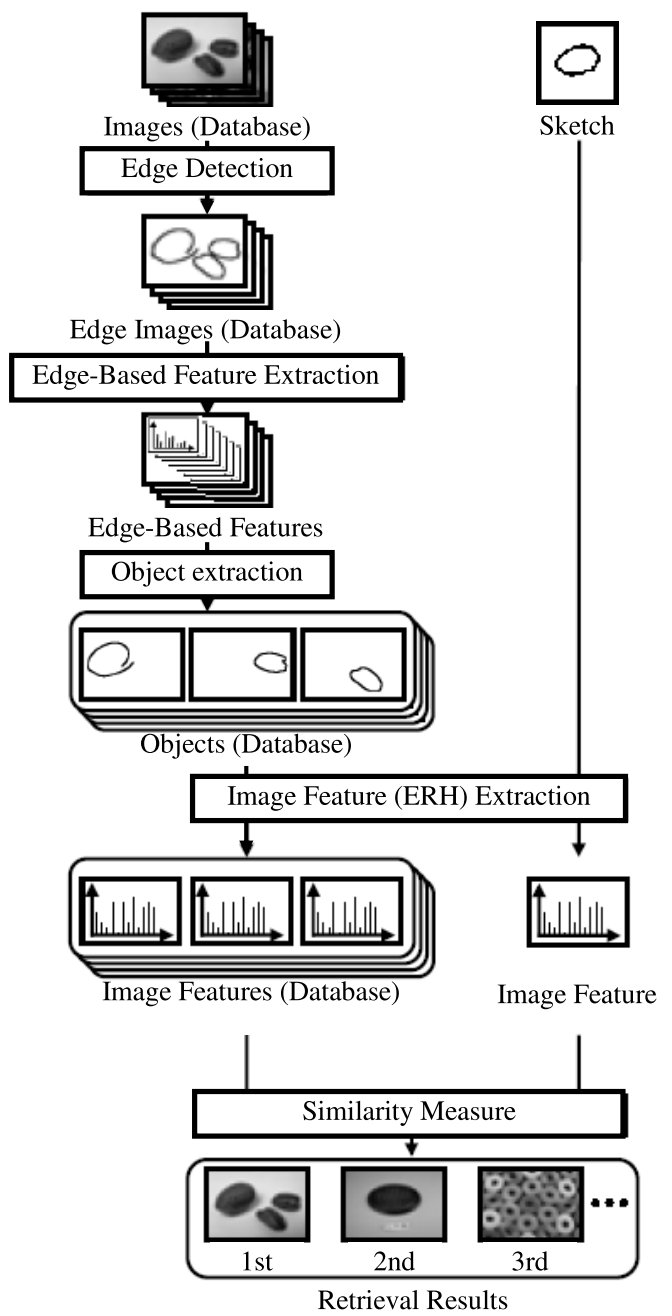

Fig. 1 Overview of query-by-sketch partial image retrieval system.

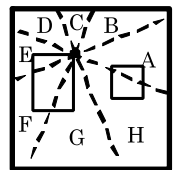

(a-1)

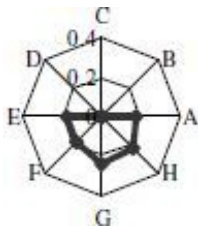

(b-1)

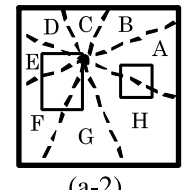

(a-2)

(a) Edge image and pixel of interest

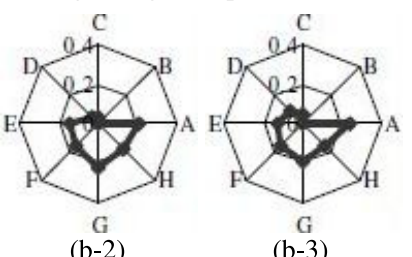

(b) Edge pixel distribution

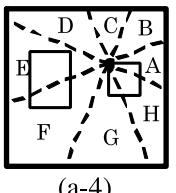

$(a-4)$

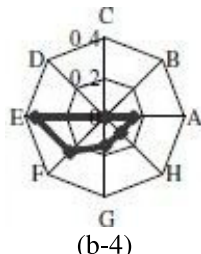

(b-4)
Fig. 2 Examples of global edge pixel distributions obtained from each pixel of interest.

Between Figs. 2(b-1) to 2(b-3), the edge pixel distribution varies continuously with the movement of the pixel of interest, but between Figs. 2(b-3) and 2(b-4), the edge pixel distribution varies greatly. Table 1 shows the similarities between Fig. 2(b-1) and (b-2), (b-2) and (b-3), (b-3) and (b-4). The features of the line pixel distributions of edge pixels $i$
Table 1 Similarity of edge pixel distributions.

\begin{tabular}{|c|c|c|c|}
\hline & $(\mathrm{b}-1) \Leftrightarrow(\mathrm{b}-2)$ & $(\mathrm{b}-2) \Leftrightarrow(\mathrm{b}-3)$ & $(\mathrm{b}-3) \Leftrightarrow(\mathrm{b}-4)$ \\
\hline Similarity & 0.910 & 0.883 & 0.671 \\
\hline
\end{tabular}

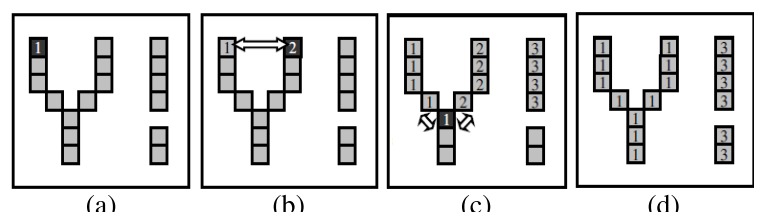

Fig. 3 Overview of object extraction using edge-based features: (a) assignment of a number, (b) comparison of the features, (c) recording of similarity, (d) result of object extraction.

and $j$ are compared by the histogram intersection, using the following degree of similarity $S_{i j}$ :

$$
S_{i j}=\sum_{k=0}^{7} \min \left(F_{i k}, F_{j k}\right),
$$

where $F_{i k}$ and $F_{j k}$ are the values of a region $k$ of the features of the edge pixels $i$ and $j$, respectively. Figure 3 shows an overview of object extraction. First, a number is assigned to the first pixel of interest, as in Fig.3(a). Next, an edge pixel that has not been assigned a number becomes the pixel of interest, and its edge-based feature is compared with that of each edge pixel that has been allocated a number. If its edge-based feature is not similar to that of any of the pixels with assigned numbers, it is assigned a new number, as in Fig. 3(b). If there is a numbered edge pixel that has a similar edge-based feature, then the pixel of interest is assigned that same number. If there are two or more edge pixels with similar edge-based features, then this is recorded, as in Fig. 3(c). The above process is performed on the edge pixels until a number has been assigned to each of them. Finally, the numbers are reassigned so that pixels that have similar edge-based features receive the same number, as in Fig. 3(d). The features of the line pixel distributions of edge pixels $i$ and $j$ are compared by Eq. (1). If the degree of similarity exceeds a threshold value, then edge pixels $i$ and $j$ are considered to be edges of the same object, and they are extracted. In this method, we begin with a high threshold value, and object extraction continues with decreasing threshold values until there is no further change in the number of extracted objects.

\section{Experimental Results}

\subsection{Object Extraction}

In order to confirm the effectiveness of this method, we used edge-based features to extract objects from images that included several objects. In these experiments, we used images from the Corel Photo Gallery collection. Examples of the results of this experiment are shown in Table 2. Two balloons were extracted, as shown in Table 2(a); an egg and a card were extracted, as shown in Table 2(b); and three paper clips were extracted, as shown in Table 2(c). From the 
Table 2 Examples of the objects extracted.

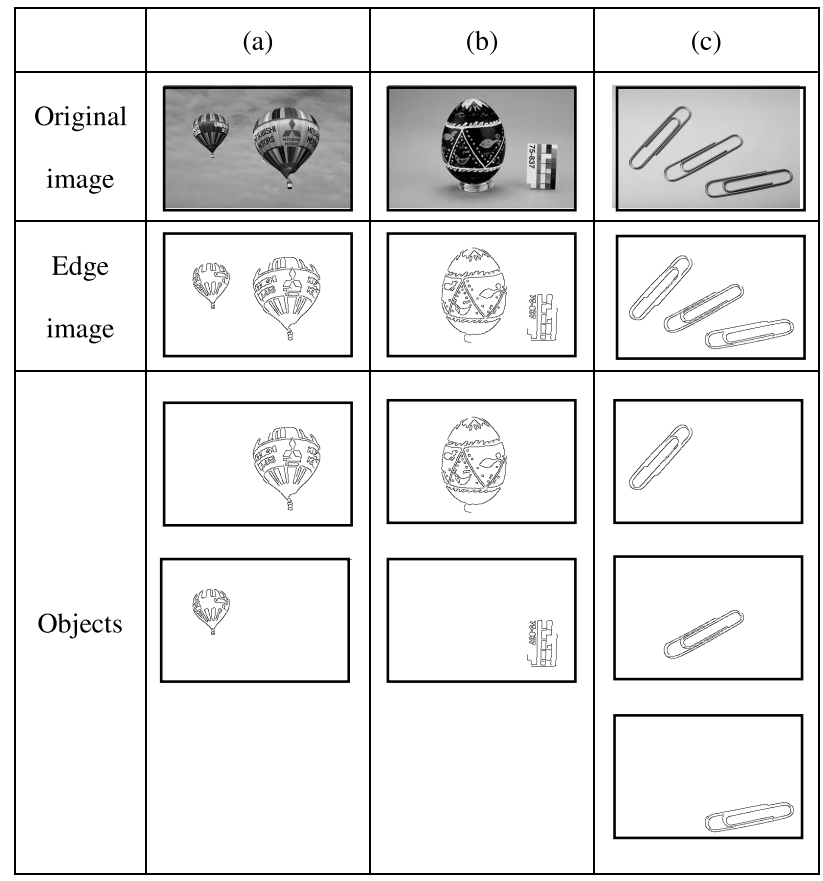

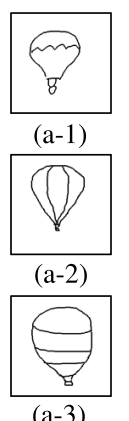

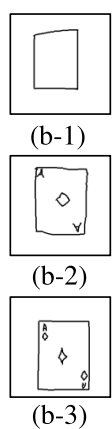

(b)

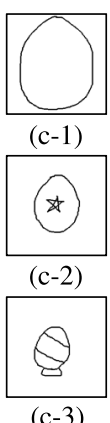

(c)

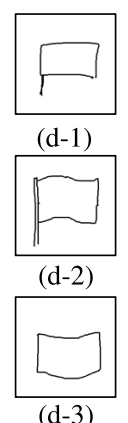

(d)

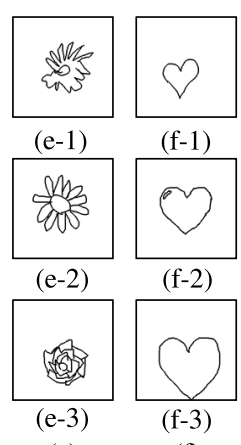

(f)
Fig. 4 Sketch of query used in retrieval experiments.

results shown in Table 2, we can confirm that this method has made it possible to extract objects from an image that contains several objects.

\subsection{Retrieval Experiments}

In order to confirm the effectiveness of this method, retrieval experiments were conducted on 20,000 images from the Corel Photo Gallery. We compared three methods: the proposed method, the ERH without object extraction, and the ERH with labeling. In the ERH with labeling, connected edge pixels are labeled with the same object. The retrieval targets were "balloon", "card", "egg", "flag", "flower", and "heart", and in the database, the relevant images included 103 for "balloon", 89 for "card", 102 for "egg", 107 for "flag", 498 for "flower", and 44 for "heart". The relevant images were categorized by majority vote in three people. The queries were drawn by three examiners other than the

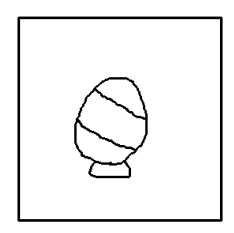

(a) Query

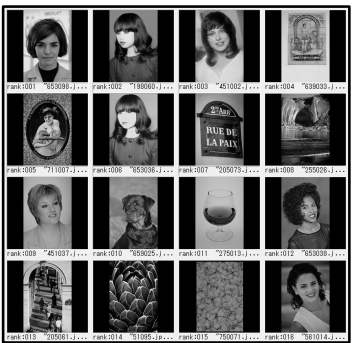

(c) Result using ERH without object extraction

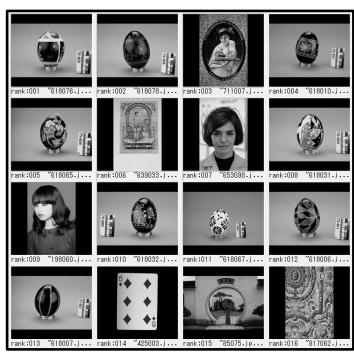

(b) Result using ERH with object extraction

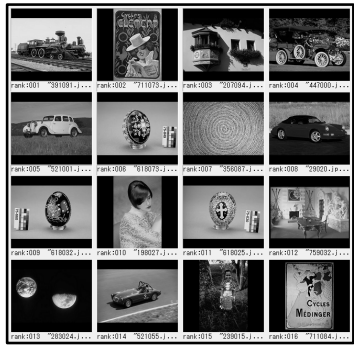

(d) Result using ERH with labeling
Fig.5 Example of retrieval results for "egg."

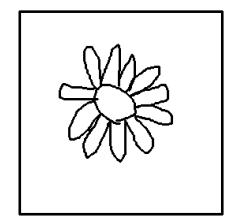

(a) Query

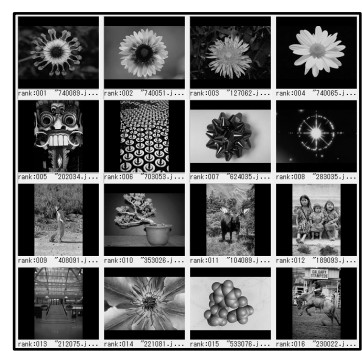

(c) Result using ERH without object extraction

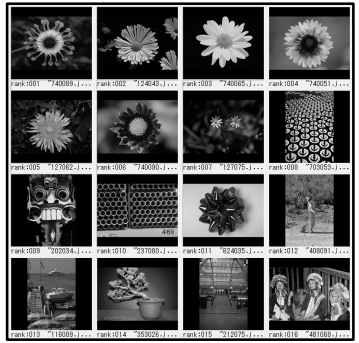
object extraction

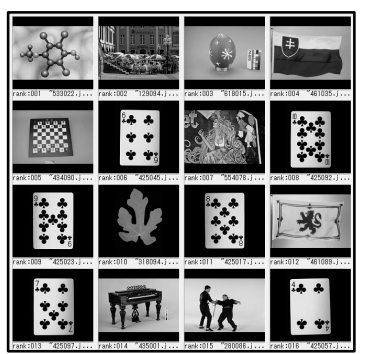

(d) Result using ERH with labeling (b) Result using ERH with

Fig. 6 Example of retrieval results for "flower."

authors, displayed in Fig. 4. The retrieval experiments for these queries used our system but were conducted by examiners. Figures 5 and 6 show the examples of the top 16 retrieval results obtained by using the retrieval targets "egg" (in Fig. 4(c-3)) and "flower" (in Fig. 4(e-2)), respectively. Figures 5 and 6 show that the ERH with object extraction retrieved a greater number of relevant images than did the other methods. In Fig. 5, because the number of the relevant images of an "egg" which have "egg" and another object 
is 101 in 102, we can confirm that using object extraction worked effectively. On the other hand, in Fig. 6, the proposed method retrieved images which have a "flower" and other objects (2nd and 7th ranked images). We note that it may be that labeling cannot be used to extract an object that is configured from a number of edge curves.

We evaluated the results quantitatively by using Mean Average Precision (MAP). MAP is a mean of Average Precision (AP), which is defined as follows:

$$
A P=\frac{1}{N} \sum_{i=1}^{N} P_{i},
$$

where $P_{i}$ is the precision of the $i$ th relevant image, and $N$ is the number of relevant images included in the database. Precision is the proportion of the retrieved images that are relevant. Precision $p(T)$ are defined as follows:

$$
p(T)=\frac{n(T)}{T},
$$

where $T$ is the number of images retrieved, $n(T)$ is the number of images retrieved that are included within the $T$ topranking images. In this study, we calculated MAP in each retrieval target (e.g., for "balloon", MAP was calculated by results used queries in Figs. 4(a-1)-(a-3).), and plotted MAP

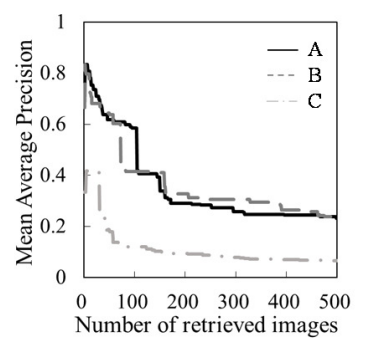

(a) "balloon"

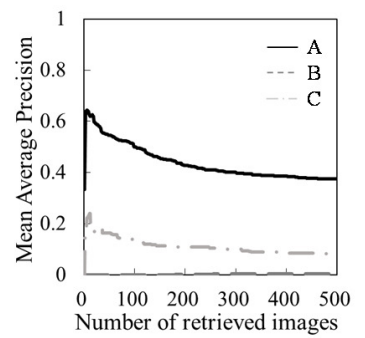

(c) "egg"

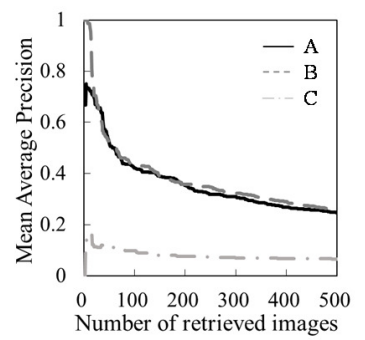

(e) "flower"

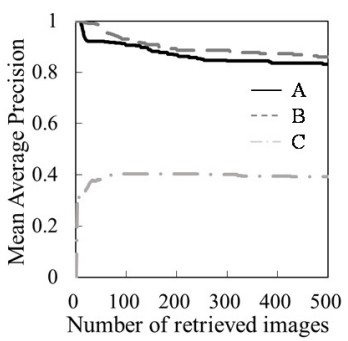

(b) "card"

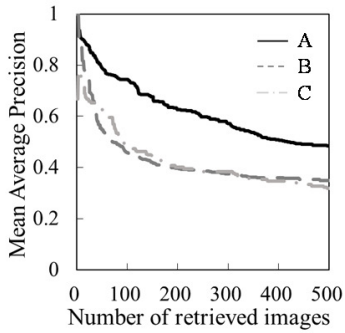

(d) "flag"

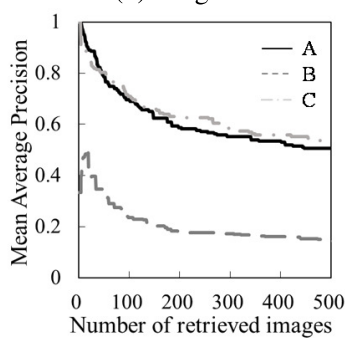

(f) "heart"
Fig. 7 Mean Average Precision of the retrieved images. A indicates ERH with object extraction, $\mathrm{B}$ indicates ERH without object extraction, and $\mathrm{C}$ indicates ERH with labeling. of each number of retrieved images. The higher the MAP, the better the accuracy of the retrieval method. The MAP of the results using queries of Fig. 4 are shown in Fig. 7. Figure 7 shows that the proposed method is more effective than the previous method in case of target of "egg", "flag", and "heart", as same as that in case of target of "balloon" and "flower", less effective than that in case of "card". Altogether, the proposed method is effective. The reason why the pattern which the proposed method is not effective is because object extraction caused over-partition of object edge, hence false retrievals were introduced.

\section{Conclusion}

In this study, we proposed an object extraction method that uses edge-based features in order to enable the retrieval of partial images by a query-by-sketch image retrieval system. We conducted retrieval experiments using 20,000 images from the Corel Photo Gallery collection, and evaluated the method qualitatively by examining the top-ranking images in the retrieval results, and quantitatively by evaluating the precision-recall graphs and the average precision. We confirmed that the retrieval accuracy was improved by using object extraction based on edge-based features, and this enabled the retrieval of partial images by the query-by-sketch image retrieval system.

This work was supported by the Ministry of Education, Culture, Sports, Science and Technology of Japan under grant number 26330135.

\section{References}

[1] D. Forsyth, J. Malik, and R. Wilensky, "Searching for digital pictures," Scientific American, vol.276, no.6, pp.88-93, 1997.

[2] R. Datta, D. Joshi, J. Li, and J.Z. Wang, "Image retrieval: Ideas, influences, and trends of the new age," ACM Computing Surveys, vol.40, no.2, article 5, pp.1-60, 2008.

[3] C.S. Won, D.K. Park, and S.-J. Park, "Efficient use of MPEG-7 edge histogram descriptor," Etri Journal, vol.24, no.1, pp.23-30, 2002.

[4] A. Chalechale, A. Mertins, and G. Naghdy, "Edge image description using angular radial partitioning," Proc. IEE Vision, Image and Signal Processing, vol.151, no.2, pp.93-101, 2004.

[5] M. Eitz, K. Hildebrand, T. Boubekeur, and M. Alexa, "A descriptor for large scale image retrieval based on sketched feature lines," Symposium on Sketch-Based Interfaces and Modeling, pp.29-36, 2009.

[6] Y. Cao, C. Wang, L. Zhang, and L. Zhang, "Edgel index for largescale sketch-based image search,” IEEE Conf. CVPR, pp.761-768, 2011.

[7] G. Ohashi and Y. Shimodaira, "Edge-based feature extraction method and its application to image retrieval," J. Systemics, Cybernetics and Informatics, vol.1, no.5, pp.25-28, 2003.

[8] Y. Kumagai and G. Ohashi, "Query-by-sketch image retrieval using edge relation histogram," IEICE Trans. Inf. \& Syst., vol.E96-D, no.2, pp.340-348, Feb. 2013.

[9] M. Eitz, K. Hildebrand, T. Boubekeur, and M. Alexa, "Sketch-based image retrieval: Benchmark and bag-of-features descriptors," IEEE Trans. Vis. Comput. Graphics, vol.17, no.11, pp.1624-1636, 2011.

[10] A. Shrivastava, T. Malisiewicz, A. Gupta, and A.A. Efros, "Datadriven visual similarity for cross-domain image matching," ACM Transactions on Graphics, vol.30, no.6, pp.154:1-154:10, 2011. 